\title{
Agressividade diferencial de espécies do complexo Fusarium graminearum em interação com o fungicida tebuconazole na redução do rendimento de trigo
}

\author{
Differential aggressiveness of species of the Fusarium graminearum complex in interaction with \\ tebuconazole fungicide on the reduction of wheat grain yield
}

\author{
Pierri Spolti ${ }^{\mathrm{I}}$ Emerson Medeiros Del Ponte ${ }^{\mathrm{I}^{*}}$
}

\section{RESUMO}

No Brasil, a giberela em trigo é causada por espécies do complexo Fusarium graminearum, especialmente F. graminearum sensu stricto (Fgss) e F. meridionale (Fmer), as quais variam quanto ao potencial toxigênico. O objetivo deste trabalho foi avaliar a relação entre características fenotípicas e agressividade dessas duas espécies associadas ao uso do fungicida tebuconazole, da classe dos triazóis, com a redução de rendimento do trigo. Em dez isolados Fgss e nove Fmer, foram avaliados: esporulação total, taxa de germinação e sensibilidade ao fungicida tebuconazole. A inoculação, para cada isolado, foi feita por aspersão em espigas e as variáveis severidade da doença, incidência de grãos giberelados e o peso de grãos foram avaliados. $O$ efeito de tebuconazole na redução da doença foi avaliado em ação protetora, seguida de inoculação na espigueta central de plantas da cultivar 'BRS Guamirim', com os seguintes tipos de inóculo: somente Fgss, somente Fmer ou a mistura de ambos (1:1). Isolados Fgss apresentaram maior esporulação total, maior taxa de germinação e foram menos sensíveis ao tebuconazole em comparação a Fmer. Grãos giberelados por isolados Fmer apresentaram 50\% maior peso do que aqueles provenientes de inoculações com Fgss. O tebuconazole apresentou efeito fungistático e os grãos de espigas tratadas com o fungicida apresentaram peso $25 \%$ superior aos não tratados. Sugere-se que diferenças no potencial de dano aos grãos pelas duas espécies, assim como o efeito fungistático de triazóis, podem ajudar a explicar a co-ocorrência de diferentes micotoxinas, o que ainda necessita ser confirmado com dados de campo.

Palavras-chave: Fusarium graminearum sensu stricto, Fusarium meridionale, peso de grãos, análise multivariada.

\section{ABSTRACT}

Fusarium head blight of wheat in Brazil is caused mainly by two species of the Fusarium graminearum species complex: F. graminearum sensu stricto (Fgss) and F. meridionale (Fmer), which vary in relation to toxigenic potential. The aim of this study was to evaluate the effect of phenotypic traits and aggressiveness of these two species, associated with the tebuconazole fungicide, of the triazole group, on the reduction of wheat yield. Ten Fgss strains and Fmer strains were evaluated with regards to: total sporulation, germination rate and tebuconazole sensitivity. The strains were spray-inoculated onto BRS Guamirim cv. 'plants' at full flowering and disease severity, Fusariumdamaged kernels, and kernel weight were evaluated. The effect of tebuconazole in disease control was evaluated through spraying the fungicide and then inoculating the central-floret of the wheat head with the following inoculum treatments: a mixture of all Fgss strains, a mixture of all Fmer strains or a mixture of all isolates of both species (1:1). Strains of Fgss showed higher total sporulation and germination rates and were less sensitivity to tebuconazole. The weight of Fusarium-damaged kernels from inoculations with Fmer strains was 50\% higher than those inoculated with Fgss. A fungistatic effect on the disease was found for tebuconazole application and harvested kernels showed 25\% higher grain weight than the untreated kernels. It is suggested that the distinct yield loss potential by these two species, in association with the fungistatic effect of triazoles could explain the co-occurrence of different mycotoxins in the harvested kernels, which needs to be proven with field data.

Key words: Fusarium graminearum sensu stricto, Fusarium meridionale, kernel weight, multivariate analysis.

\section{INTRODUÇÃO}

A giberela está entre as doenças de maior importância mundial na cultura do trigo. No Brasil, é causada principalmente por espécies do complexo Fusarium graminearum, com destaque para $\boldsymbol{F}$. graminearum sensu stricto [ou simplesmente $\boldsymbol{F}$.

IDepartamento de Fitossanidade, Faculdade de Agronomia, Universidade Federal do Rio Grande do Sul (UFRGS), 90001-970, Porto Alegre, RS, Brasil. E-mail: emerson.delponte@ufrgs.br. *Autor para correspondência. 
graminearum (Fgss)] e $\boldsymbol{F}$. meridionale (Fmer) (SCOZ et al., 2009;ASTOLFI et al., 2012). Os danos ocasionados por giberela em trigo no Brasil incluem a redução no rendimento e a contaminação com as micotoxinas de desoxinivalenol (DON) e nivalenol (NIV) (CASA et al., 2004; DEL PONTE et al., 2012). No Sul do Brasil, as populações de Fgss (a dominante, com $90 \%$ de frequência em isolamentos) e Fmer têm apresentado consistentemente genótipo tricoteceno15-A(acetil)DON, uma forma acetilada de DON, e NIV, respectivamente, o que sugere que podem produzir diferentes concentrações de DON e nivalenol (SCOZ et al., 2009; ASTOLFI et al., 2012). SPOLTI et al. (2012a), em um estudo com dois isolados de cada uma dessas duas espécies, observaram maior taxa de esporulação, maior agressividade e menor sensibilidade a tebuconazole em isolados Fgss comparados a Fmer. Os autores sugeriram que essas características poderiam resultar em uma maior vantagem adaptativa para Fgss. Recentemente, DEL PONTE et al. (2012) analisaram 66 amostras de grãos de trigo provenientes de lavouras comerciais do Rio Grande do Sul e observaram a ocorrência simultânea das micotoxinas DON e nivalenol em 59 amostras, com concentração média de DON igual a $540 \mu \mathrm{g} \mathrm{kg}^{-1}$ e de nivalenol igual a $337 \mu \mathrm{g} \mathrm{kg}^{-1}$, evidenciando que as populações produzem ambas as micotoxinas. Para o manejo da giberela e das micotoxinas nos grãos, é recomendado o uso de cultivares com maior grau de resistência, aplicação de fungicidas e o ajuste dos mecanismos da colheita mecânica para a eliminação de grãos giberelados (McMULLEN et al., 2012). Fungicidas do grupo dos triazóis, que possuem ação fungistática sobre $\boldsymbol{F}$. graminearum sensu lato, têm sido indicados para uso durante o florescimento pleno (YIN et al., 2009). Considerando que grãos giberelados são menores e mais leves do que grãos sadios, especialmente aqueles infectados no início do florescimento, o ajuste dos mecanismos da colheita mecânica pode facilitar a eliminação de grãos giberelados e, consequentemente, reduzir os níveis de micotoxinas nos lotes de grãos colhidos (SALGADO et al., 2011). Além da extensão da janela de infecção que pode levar a menores danos em rendimento em infecções tardias (DEL PONTE et al., 2007; COWGER \& ARRELANO, 2010), não se tem conhecimento se diferenças fenotípicas entre as espécies do complexo podem influenciar no rendimento e formação dos grãos. No presente estudo, testou-se a hipótese de que espécies dominantes obtidas de grãos giberelados possuem maior potencial de reduzir o peso de grãos do que espécies menos frequentes, especialmente sob o efeito fungistático de tebuconazole. Assim, objetivou-se: i) avaliar características fenotípicas de um conjunto de isolados das duas espécies do complexo (Fgss e Fmer), discriminando-as por meio de análise multivariada; ii) avaliar o efeito fungistático de tebuconazole no controle da doença e redução de danos em grãos de espigas inoculadas com as duas espécies.

\section{MATERIAL E MÉTODOS}

Coleção de isolados e plantio em casa de vegetação Foram utilizados 19 isolados, sendo 10

Fgss e 9 Fmer obtidos de espigas sintomáticas de lavouras de trigo e cevada em três safras (2007 a 2009) do norte do estado do Rio Grande do Sul. O isolamento, a purificação e a identificação da espécie filogenética foram realizados conforme descrito previamente (ASTOLFI et al., 2012). Esses isolados foram distintos dos quatro isolados utilizados em um estudo prévio (SPOLTI et al., 2012a). Para os estudos de agressividade, foi utilizada a cultivar 'BRS Guamirim', mesma utilizada no trabalho anterior (SPOLTI et al., 2012a) para se ter um padrão de comparação dos resultados. Dez sementes foram semeadas em vasos de $5 \mathrm{~L}$, contendo substrato 1:1:1 (húmus, solo e vermiculita) com adubação de base com NPK (10:10:10) e mantidos em casa de vegetação do plantio à maturação fisiológica dos grãos. A temperatura média da casa de vegetação durante a condução do experimento foi de $26^{\circ} \mathrm{C}$ e umidade relativa do ar $\geq 60 \%$.

\section{Caracterização fenotípica dos isolados}

Cada isolado foi caracterizado quanto à esporulação total, germinação e sensibilidade ao tebuconazole, inferida pela concentração efetiva, que reduz $50 \%$ do crescimento micelial $\left(\mathrm{CE}_{50}\right)$, conforme descrito previamente (SPOLTI et al., 2012b). A agressividade foi avaliada por inoculação por aspersão em espigas no momento da antese plena com uma suspensão de esporos $\left(10^{5}\right.$ macroconídiosmL $\left.\mathrm{L}^{-1}\right)$, aplicada até o ponto de escorrimento $\left(\approx 10 \mathrm{mLvaso}^{-1}\right)$. Cada isolado foi inoculado separadamente nessa etapa do estudo. Após a inoculação, as plantas foram mantidas a $25^{\circ} \mathrm{Ce} 100 \%$ de UR por $48 \mathrm{~h}$ (MIEDANER et al., 2003). Nesse ensaio, foi utilizada a inoculação por aspersão para mimetizar as condições naturais de infecção onde se tem eventos múltiplos de infecção e para reduzir o efeito da resistência do Tipo II (menor taxa de colonização da espiga) na reprodução dos dados. As avaliações de incidência ( $\mathrm{n}^{\circ} \mathrm{o}$ de espigas com pelo menos uma espigueta giberelada) e severidade de giberela (proporção de espiguetas gibereladas) foram 
realizadas a partir do sexto dia após a inoculação (DAI), com intervalo de quatro dias até o $18^{\circ} \mathrm{DAI}$, o que permitiu calcular a área abaixo da curva de progresso da doença (AACPD). No momento da maturação fisiológica $(\approx 43 \mathrm{DAI})$, as espigas foram trilhadas manualmente e os grãos classificados visualmente em sadios e giberelados (DEL PONTE et al., 2012).

Interação fungicida $\mathrm{x}$ isolados

Em outro experimento, plantas da cv. 'BRS Guamirim' foram aspergidas na antese plena com um fungicida tebuconazole (Tebuconazole Nortox 200 EC, Nortox S.A.) na dose de $0,75 \mathrm{~L}$ p.c. ha ${ }^{-1}$ e mantidas em casa de vegetação por $48 \mathrm{~h}$. O produto comercial utilizado neste estudo não possui registro no Brasil para o controle de giberela em trigo e, portanto, sua utilização, neste trabalho, não configura indicação de uso comercial (MAPA, 2013). No entanto, o tebuconazole é amplamente utilizado na América do Norte para o controle de giberela desde meados da década de 90 (McMULLEN et al., 2012). Após 48h à pulverização com o fungicida, a espigueta central de cada espiga foi inoculada conforme SPOLTI et al. (2012a). Nesse experimento, o inóculo foi obtido do mesmo conjunto de 19 isolados. No entanto, para cada espécie inoculada (Fgss e Fmer), foi obtida uma suspensão composta por partes iguais (v/v) a partir de suspensão de esporos ajustadas para cada isolado. Adicionalmente, um terceiro tratamento de inoculação constou da mistura de inóculo das duas espécies (1:1, $\mathrm{v} / \mathrm{v})$. Após a inoculação, as plantas foram mantidas a $25^{\circ} \mathrm{C}$ e $100 \%$ UR por $48 \mathrm{~h}$. A incidência e severidade foram avaliadas a partir do quinto dia à inoculação (5DAI), com intervalo de três dias até o 14DAI. No momento de maturação fisiológica ( $\approx 44 \mathrm{DAI})$, as espigas foram trilhadas manualmente e os grãos classificados visualmente em sadios ou giberelados e pesados separadamente.

Delineamento experimental e análises estatísticas

Os ensaios em casa de vegetação foram conduzidos em blocos casualizados e os experimentos in vitro em delineamento inteiramente casualizado. Nos primeiros, cada repetição foi composta por um vaso contendo 10 espigas cada e, no segundo, cada repetição foi composta por uma placa de Petri. Em ambos os casos, foram utilizadas cinco repetições. Os ensaios de esporulação, germinação e sensibilidade ao fungicida foram repetidos duas vezes e os dados agrupados, uma vez que não houve diferença entre os experimentos na análise de variância $(\alpha>0,05)$. $\mathrm{Na}$ análise multivariada, com os dados de todas as variáveis na caracterização de cada isolado, foi usado um teste de permutação para avaliar a significância da variância entre os isolados, a partir de uma matriz de semelhança estabelecida pela distância Euclidiana entre as unidades amostrais (TORRES et al., 2010). As médias das variáveis independentes foram discriminadas por pareamento e, quando significativas, foi realizado contraste ortogonal conforme a espécie filogenética (Fgss x Fmer), definindo a significância do contraste com 10.000 permutações (PILLAR \& ORLÓCI, 1996). Análise de agrupamento de todos os isolados foi utilizada considerando conjuntamente os valores médios de todas as variáveis observadas. Análise de correlação (coeficiente de Pearson) foi utilizada para definir a semelhança entre as variáveis e o índice de Gower entre as unidades amostrais. Foi adotado o critério de Ward, tomando-se como ponto de seleção (número de grupos) a significância obtida pela auto reamostragem (10.000 permutações), assumindo-se nitidez do agrupamento, quando $\alpha \geq 0,05$ (PILLAR, 1999). A análise de componentes principais foi realizada para identificar, das variáveis utilizadas no agrupamento, aquelas com maior influência na caracterização dos isolados, extraindo os fatores com autovalores $\geq 1$. As análises de permutação, agrupamento e componentes principais foram realizadas no programa MULTIV versão 2.4 (PILLAR, 2006). No experimento com a aplicação protetora de tebuconazole e inoculação por flor-única, foi realizada análise de variância, utilizando o PROC MIXED (SAS Institute, Cary, NC). Os blocos foram considerados como efeito aleatório e os fatores tipo de inóculo, a aplicação do fungicida e o tempo como efeitos fixos. As médias dos efeitos fixos foram discriminadas pela diferença mínima significativa (DMS, $\alpha=0,05$ ).

\section{RESULTADOS E DISCUSSÃO}

As características fenotípicas relacionadas à reprodução assexual e sensibilidade ao fungicida distinguiram os isolados segundo a espécie filogenética (Tabela 1). As médias de esporulação total, taxa de germinação e $\mathrm{CE}_{50}$ do fungicida tebuconazole foram as mais altas nos isolados Fgss $(\mathrm{P} \leq 0,016)$. Na média, a $\mathrm{CE}_{50}$ de Fgss foi 4,5 vezes superior à $\mathrm{CE}_{50}$ de Fmer ratificando resultados anteriores (SPOLTI et al., 2012b). Não foi possível discriminar as duas espécies quanto ao progresso da doença, tanto pela incidência como pela severidade $(\mathrm{P} \geq 0,0829)$ (Tabela 1$)$. Também não foi observada diferença entre as duas espécies em relação ao $\mathrm{n}^{\mathrm{o}}$ de grãos sadios, $\mathrm{n}$ o de grãos giberelados por espigas e peso de grãos sadios $(\mathrm{P} \geq 0,471)$. No

Ciência Rural, v.43, n.9, set, 2013. 
Tabela 1 - Análise de variância por testes de permutação de variáveis fenotípicas, relacionadas ao progresso da doença e aos danos após a inoculação por aspersão de 10 isolados Fusarium graminearum sensu stricto (Fgss) e nove isolados Fusarium meridionale (Fmer).

\begin{tabular}{|c|c|c|c|c|}
\hline \multirow{2}{*}{ Variável } & \multicolumn{2}{|c|}{--------------Valores médios ${ }^{(1)}$} & \multirow{2}{*}{$\mathrm{S} . \mathrm{Q}^{(2)}$} & \multirow{2}{*}{$\mathrm{P}>\mathrm{F}^{(3)}$} \\
\hline & Fgss & Fmer & & \\
\hline \multicolumn{5}{|l|}{ Característica fenotípica } \\
\hline $\begin{array}{l}\text { Esporulação } \\
\left(\text { macroconídios } / \mathrm{mLx} 10^{3} \text { ) }\right.\end{array}$ & 21,91 & 12,68 & 1220000000 & 0,0166 \\
\hline Germinação (\%) & 34,93 & 8,07 & 1210000000 & 0,0146 \\
\hline CE50 Tebuconazole $\left(\mu \mathrm{LmL}^{-1}\right)$ & 0,259 & 0,057 & 0,58432 & 0,0001 \\
\hline \multicolumn{5}{|l|}{ Progresso da doença ${ }^{(4)}$} \\
\hline $\mathrm{AACPD}_{\text {(incidência) }}$ & 758 & 937 & 3338000 & 0,0829 \\
\hline $\mathrm{AACPD}_{\text {(severidade) }}$ & 221 & 255 & 71188 & 0,3273 \\
\hline \multicolumn{5}{|l|}{ Danos em produtividade } \\
\hline № grãos sadios/espiga & 8,43 & 7,49 & 12,65 & 0,5250 \\
\hline № grãos giberelados/espiga & 12,17 & 12,73 & 4,35 & 0,7149 \\
\hline Peso de grãos sadios (g) & 0,0335 & 0,0353 & 0,0000467 & 0,4708 \\
\hline Peso de grãos giberelados (g) & 0,0143 & 0,0269 & 0,00225 & 0,0001 \\
\hline
\end{tabular}

${ }^{(1)}$ Valores médios de 10 isolados Fgss e nove isolados Fmer após o agrupamento pelo genótipo tricoteceno. ${ }^{(2)}$ Soma de quadrado indica a variância entre os isolados. ${ }^{(3)}$ Valor de $P$ estimado após 10.000 permutações. ${ }^{(4)}$ Área abaixo da curva de progresso da incidência e da severidade.

entanto, espigas inoculadas com Fgss tiveram grãos assintomáticos com peso $57 \%$ maior do que os grãos giberelados, enquanto que com Fmer, essa diferença foi de 24\% (Tabela 1). Grãos giberelados por isolados Fmer tiveram um peso $47 \%$ superior ao de grãos giberelados por isolados Fgss $(\mathrm{P}<0,001)$. Na análise de componentes principais, os três primeiros eixos apresentaram significância estatística $(\mathrm{P}<0,05)$ e autovalores superiores a 1. Somadas as variâncias, os três primeiros eixos representam uma variância acumulada de 44,26\%. Dentro dos eixos 1 a 3 , as variáveis $\mathrm{n}^{-}$de grãos assintomáticos/espigas, peso de grãos giberelados e esporulação, apresentaram os maiores autovetores (valores absolutos), sendo responsáveis por $48 \%, 34 \%$ e $32 \%$ da variância nos eixos 1 a 3 , respectivamente. Pela análise multivariada, não foi possível discriminar as espécies filogenéticas. $\mathrm{Na}$ análise de agrupamento foram identificados três grupos nítidos $(\mathrm{P}>0,05)$, com 12,5 e 2 isolados em cada grupo composto por isolados de ambas as espécies. A incapacidade de discriminar as espécies nesse estudo pode ser devida à metodologia de inoculação por aspersão e ao papel das micotoxinas como fatores de virulência (MIEDANER et al., 2003). Dados da literatura mostram que DON tem papel na colonização, mas não é necessário para causar as infecções (PURI \& ZHONG, 2010; SPOLTI et al., 2012a). Pela inoculação na espigueta central, foi evidenciado o efeito das espécies, da aplicação ou não do fungicida e do tempo na incidência e na severidade da doença $(\mathrm{P}<0,0001)$. Com exceção da interação tripla, todas as demais interações foram significativas $(\mathrm{P} \leq 0,046)$. Sem o fungicida, houve diferença entre a espécie inoculada na incidência de giberela apenas na avaliação realizada 5DAI, sendo menor nas plantas inoculadas com Fmer do que nos demais tratamentos (Figura 1). Na ausência do fungicida, a severidade foi inferior nos tratamentos com Fmer em comparação à Fgss ou a mistura. Sem o fungicida, a severidade de giberela com a inoculação de Fgss, mistura e Fmer foi de $80 \%, 82 \%$ e $38 \%$, respectivamente (Figura 1). O tebuconazole apresentou efeito fungistático, indiferente à espécie inoculada, promovendo um atraso no desenvolvimento da doença nas espigas, mantendo a severidade em valores inferiores a 5\% até 11 DAI. Aos 14 DAI, no entanto, a severidade da 


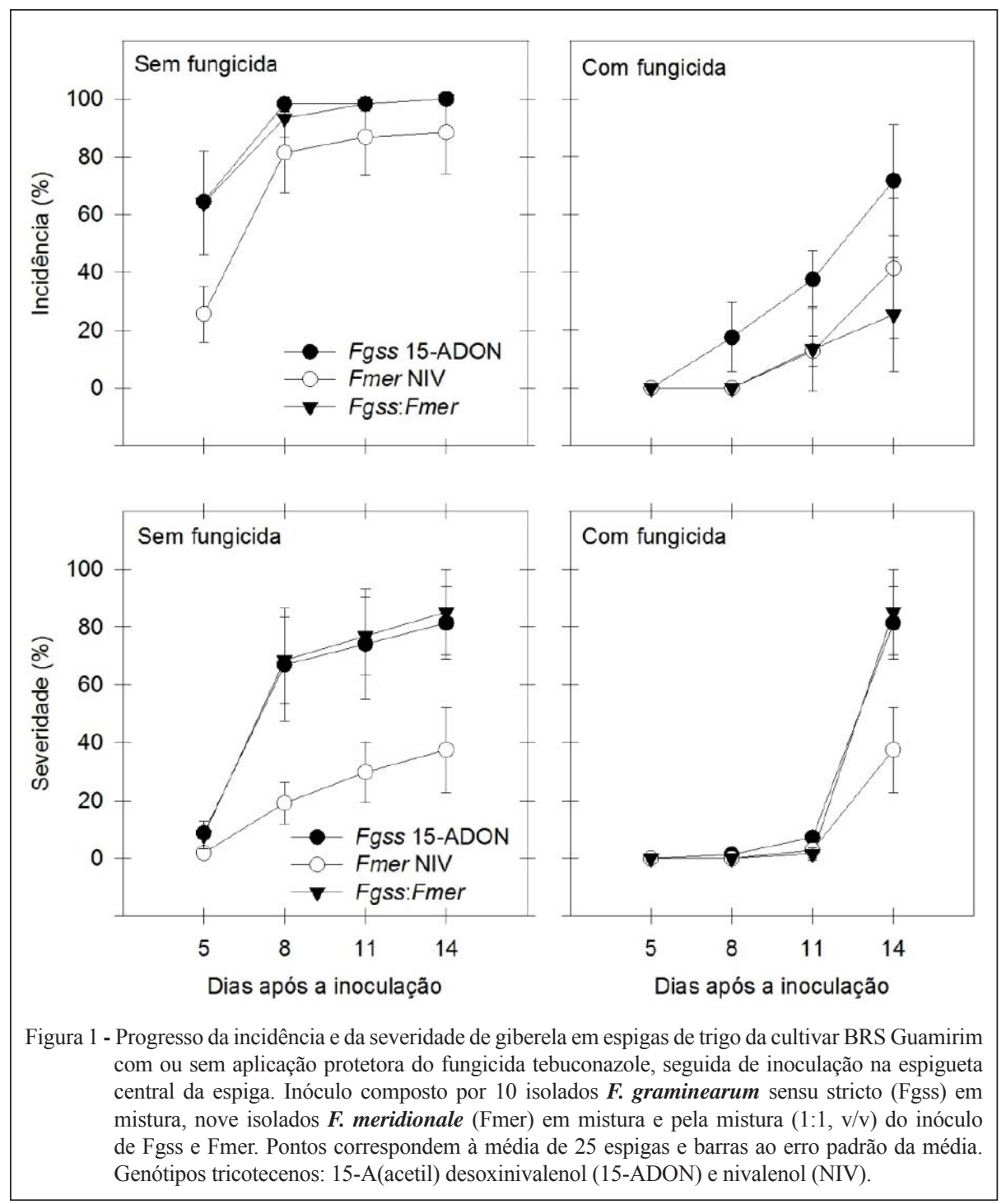

doença atingiu valores similares ao observado sem a aplicação do fungicida (Figura 1). Foi observada evidência do efeito da espécie na incidência de grãos giberelados com a aplicação ou não do fungicida tebuconazole, com maior incidência de grãos giberelados pela inoculação de Fgss e mistura em relação à Fmer. A aplicação do fungicida não reduziu a incidência de grãos giberelados, indiferente à espécie inoculada (Tabela 2). Independente da aplicação de tebuconazole, grãos giberelados por Fmer apresentaram peso superior àqueles giberelados por Fgss. Finalmente, indiferente à espécie inoculada, grãos giberelados de espigas tratadas com o fungicida apresentaram peso superior aos grãos giberelados sem o tratamento (Tabela 2). Relatos contraditórios do efeito de tebuconazole na redução de grãos giberelados são citados na literatura, o que pode ser devido à diversidade de metodologias empregadas e pela complexidade envolvida na eficiência do fungicida que envolve o momento de aplicação, agressividade dos isolados e nível de resistência da cultivar (MESTERHÁZY et al., 2003; PAUL et al., 2005). A relação entre grãos giberelados e níveis de tricotecenos em lotes de grãos é complexa e envolve fatores ambientais e agronômicos (PAUL et al., 2005). Observou-se no presente estudo que, além da incidência de grãos giberelados, as duas espécies ocorrentes em trigo no Brasil têm potencial distinto de causar danos no rendimento, o que pode influenciar nos níveis de micotoxinas encontradas em grãos da mesma região onde os isolados foram obtidos. Por meio de ajustes da velocidade da corrente de ar, para a seleção e limpeza dos grãos, é possível excluir grãos com menor peso e/ou mal formados (SALGADO et

Ciência Rural, v.43, n.9, set, 2013. 
Tabela 2 - Incidência e peso dos grãos giberelados oriundos de espigas de trigo tratadas ou não com tebuconazole 24 h prévias à inoculação. Inóculo composto por isolados de espécies do complexo F. graminearum e aplicado na espigueta central de plantas da 'cultivar' BRS Guamirim.

\begin{tabular}{|c|c|c|c|c|}
\hline \multirow{2}{*}{ Inóculo ${ }^{(1)}$} & \multicolumn{2}{|c|}{--------- Grãos giberelados $(\%)^{(2)}$} & \multicolumn{2}{|c|}{--------- Peso de grãos giberelados (g) ----- } \\
\hline & Sem fungicida & Com fungicida & Sem fungicida & Com fungicida \\
\hline Fgss & 37,4 & $33,1^{\text {ns }}$ & 0,016 & $0,022 *(4)$ \\
\hline Fmer & 20,2 & 18,6 & 0,023 & $0,028^{*}$ \\
\hline Fgss + Fmer & 34,7 & 30,1 & 0,015 & $0,023 *$ \\
\hline $\mathrm{DMS}^{(3)}$ & 5,7 & 7,4 & 0,005 & 0,003 \\
\hline
\end{tabular}

${ }^{(1)} 10$ isolados $\boldsymbol{F}$. graminearum sensu stricto (Fgss), nove isolados $\boldsymbol{F}$. meridionale (Fmer) ou mistura 1:1 (Fgss: Fmer). ${ }^{(2)}$ grãos com aspecto esbranquiçado e/ou com a presença de sinais do patógeno. ${ }^{(3)}$ Diferença mínima significativa para a discriminação das médias entre os inóculos. ${ }^{\text {ns }}=$ não significativo. ${ }^{(4)}$ Significância da comparação das médias dentro do tratamento com o mesmo inóculo. Ex.: peso de grãos inoculados com Fgss vs. peso de grãos inoculados com Fgss de espigas pulverizadas com tebuconazole.

al., 2011). Considerando que grãos infectados por isolados Fmer apresentam peso até 50\% superior aos infectados por Fgss, os primeiros podem ter maiores chances de permanecer no lote enquanto que grãos giberelados por Fgss poderiam ter maior chance de descarte, permanecendo no campo e contribuindo para o inóculo local (INCHet al., 2003). Essa hipótese, no entanto, deve ser testada em condições de campo.

\section{CONCLUSÃO}

Indiferente às diferenças fenotípicas entre F. graminearum e $\boldsymbol{F}$. meridionale, não foi possível discriminar as duas espécies pela análise multivariada pela inoculação por aspersão. Entre as duas espécies estudadas, F. graminearum mostrou mais alto potencial de redução de rendimento, indiferente ao método de inoculação. O efeito fungistático de tebuconazole reduz os níveis de doença e os danos causados por giberela, indiferente à espécie filogenética, mas pode favorecer a retenção de grãos infectados com maior peso e potencialmente contaminados com micotoxinas.

\section{REFERÊNCIAS}

ASTOLFI, P. et al. Genetic population structure and trichothecene genotypes of Fusarium graminearum isolated from wheat in southern Brazil. Plant Pathology, v.61, p.289-295, 2012. Dísponível em: <http://onlinelibrary.wiley.com/doi/10.1111/ j.1365-3059.2011.02515.x/abstract>. Acesso em: 15 ago. 2011.

CASA, R.T. et al. Danos causados pela infecção de Gibberella zeae em trigo. Fitopatologia Brasileira, v.29, p.289-293, 2004. Disponível em: <http://www.scielo.br/pdf/fb/v29n3/20363.pdf>. Acesso em: 10 jul. 2011.

COWGER, C.; ARRELlANO, C. Plump kernels with high deoxynivalenol linked to late Gibberella zeae infection and marginal disease conditions in winter wheat. Phytopathology, v.100, p.719728, 2010. Disponível em: <http://apsjournals.apsnet.org/doi/ abs/10.1094/PHYTO-100-7-0719>. Acesso em: 5 fev. 2011.

DEL PONTE, E.M. et al. Influence of growth stage on Fusarium head blight and deoxynivalenol production in wheat. Journal of Phytopathology, v.155, p.577-581, 2007. Disponível em: <http:// onlinelibrary.wiley.com/doi/10.1111/j.1439-0434.2007.01281.x/ abstract>. Acesso em: 26 jul. 2010.

DEL PONTE, E.M. et al. Deoxynivalenol and nivalenol in commercial wheat grain related to Fusarium head blight epidemics in southern Brazil. Food Chemistry, v.132, p.1087-1091, 2012. Disponível em: <http:/www.sciencedirect.com/science/article/pii/ S0308814611016177>. Acesso em: 19 jul. 2011.

INCH, S.A.; GILBERT, J. Survival of Gibberella zeae in Fusarium-damaged wheat kernels. Plant Disease, v.87, p. 282287, 2003. Disponível em: <http://apsjournals.apsnet.org/doi/ pdf/10.1094/PDIS.2003.87.3.282>. Acesso em: 23 jan. 2010.

MAPA. Sistema de agrotóxicos fitossanitários (Agrofit). Disponível em: <http://extranet.agricultura.gov.br/agrofit_cons/ principal_agrofit_cons $>$. Acesso em: 03 mar. 2013.

MESTERHÁZY, Á. et al. Influence of wheat cultivar, species of Fusarium, and isolate aggressiveness on the efficacy of fungicides for control of Fusarium head blight. Plant Disease, v.87, p.11071115, 2003. Disponível em: <http://apsjournals.apsnet.org/doi/ pdf/10.1094/PDIS.2003.87.9.1107>. Acesso em: 12 jan. 2010.

McMULLEN, M. et al. A unified effort to fight an enemy of wheat and barley: Fusarium head blight. Plant Disease, v.96, p.17121728, 2012. Disponível em: <http://apsjournals.apsnet.org/doi/ abs/10.1094/PDIS-03-12-0291-FE>. Acesso em: 20 nov. 2012.

MIEDANER, T. et al. Comparison of spray and point inoculation to assess resistance to Fusarium head blight in a multi-environment wheat trial. Phytopathology, v.93, p.1068-1072, 2003. Disponível em: <http://apsjournals.apsnet.org/doi/pdf/10.1094/ PHYTO.2003.93.9.1068>. Acesso em: 12 jan. 2010.

PAUL, P.A. et al. Relationship between visual estimates of Fusarium head blight intensity and deoxinivalenol accumulation in harvested wheat grain: a meta-analysis. Phytopathology, v.95, 
p.1225-1236, 2005. Disponível em: <http://apsjournals.apsnet.org/ doi/abs/10.1094/PHYTO-95-1225>. Acesso em: 12 jan. 2010.

PILLAR, V.P.; ORLÓCI, L. On randomization testing in vegetation science: multifactor comparisons of relevant groups. Journal of Vegetation Science, v.7, p.585-592, 1996. Disponível em: <http:// www.ecologia.ufrgs.br/ adrimelo/div/Pillar_Orloci_1996_JVS. pdf $>$. Acesso em: 20 ago. 2010.

PILLAR, V.P. How sharp are classifications? Ecology, v.80, p.2508-2116, 1999. Disponível em: <http://www.jstor.org/ stable/177236>. Acesso em: 20 ago. 2010.

PILLAR, V.P. MULTIV Multivariate Exploratory Analysis, Randomization Testing and Bootstrap Resampling. User's Guide, v. 2.4. Porto Alegre: Departamento de Ecologia, Universidade Federal do Rio Grande do Sul, 2006. 51p.

PURI, K.D.; ZHONG, S. The 3ADON population of Fusarium graminearum found in North Dakota is more aggressive and produces a higher level of DON than the prevalent 15ADON population in spring wheat. Phytopathology, v.100, p.10071014, 2010. Disponível em: <http://apsjournals.apsnet.org/doi/ abs/10.1094/PHYTO-12-09-0332>. Acesso em: 13 maio, 2011.

SALGADO, J.D. et al. Grain harvesting strategies to minimize grain quality losses due to Fusarium head blight in wheat. Plant Disease, v.95, p.1448-1457, 2011. Disponível em: <http:// apsjournals.apsnet.org/doi/pdf/10.1094/PDIS-04-11-0309>. Acesso em: 20 jan. 2012
SCOZ, L.B. et al. Trichothecene mycotoxin genotypes of Fusarium graminearum sensu stricto and Fusarium meridionale in wheat from Southern Brazil. Plant Pathology, v.58, p.344-351, 2009. Disponível em: <http://onlinelibrary.wiley.com/doi/10.1111/ j.1365-3059.2008.01949.x/abstract>. Acesso em: 11 jan. 2010.

SPOLTI, P. et al. Phenotypic and pathogenic traits of two species of the Fusarium graminearum complex possessing either 15-ADON or NIV genotype. European Journal of Plant Pathology, v.133, p.621-629, 2012a. Disponível em: <http://link.springer.com/articl e/10.1007\%2Fs10658-012-9940-5>. Acesso em: 20 maio, 2012.

SPOLTI, P. et al. Sensitivity of Fusarium graminearum causing head blight of wheat in Brazil to tebuconazole and metconazole fungicides. Tropical Plant Pathology, v.37, p.419-423, 2012 b. Disponível em: <http://www.scielo.br/pdf/tpp/v37n6/a07v37n6. pdf $>$. Acesso em: 13 mar. 2013.

YIN, Y. et al. Characterization of sterol demethylation inhibitor resistant isolates of Fusarium asiaticum and F. graminearum collected from wheat in China. Phytopathology, v.99, p.487497, 2009. Disponível em: <http://apsjournals.apsnet.org/doi/ abs/10.1094/PHYTO-99-5-0487>. Acesso em: 28 out. 2011.

TORRES, O.S. et al. Properties of a randomization test for multifactor comparisons of groups. Journal of Statistical Computation and Simulation, v.80, p.1131-1150, 2010. Disponível em: <http://www.tandfonline.com/doi/abs/10.1080/00 949650902984430\#preview>. Acesso em: 15 ago. 2011. 